COMPUTATIONAL METHODS IN APPLIED MATHEMATICS, Vol.1(2001), No.1, pp.125-131

(C) Institute of Mathematics of the National Academy of Sciences of Belarus

\title{
ON THE SATURATION ASSUMPTION AND HIERARCHICAL A POSTERIORI ERROR ESTIMATOR
}

\author{
A. AGOUZAL \\ Maply. U.M.R 5558 \\ Bat. 101, Univresité Lyon1, 43 Bd 18 Novembre 1918. 69 622, villeurbanne cedex. France \\ E-mail: agouzal@maply.univ-lyon1.fr
}

\begin{abstract}
The saturation assumption is widely used in a posteriori error analysis of finite element methods. It asserts, in its simplest form, that the solution can be approximated asymptotically with quadratic finite elements than with linear ones. In this paper, we show that a simple modification of this "hypothesis" is valid, and the proof of a posteriori error estimators by several authors becomes rigoruous with this simple modification.
\end{abstract}

2000 Mathematics Subject Classification: 65N30.

Keywords: saturation assumption, hierarchical spaces, a posteriori error estimators.

\section{Introduction}

The classical proof of equivalence of some a posteriori error estimators with the energy error requires the saturation assumption [2,3]: this means, in its simplest form, that the solution can be approximated asymptotically with quadratic finite elements than with linear ones. The saturation assumption was shown to be superfluous by Nochetto in [5] for BankWeiser estimators, howeover, removing this assumption requires comparison with residual estimators. More recently W. Dorfler and R.H. Nochetto [4] have given a sufficient condition for the validity of the saturation assumption, more precisely, they have proved that "small data oscillation implies the saturation assumption"; the technique used in the last paper was taken from the a posteriori error analysis and comparison with residual estimators. Recently the author of [1], have given a direct proof of the reliability and efficiency of the hierarchical estimator without comparison with the residual estimator or the saturation assumption for conforming and nonconforming approximations.

In this paper, we show that a simple modification of this "assumption" is valid, and the proof of a posteriori error estimates of several authors is rigorous with this simple modification. As an example, we give "elementary" and direct proof of the efficiency and reliability of the hierarchical a posteriori error estimators. 


\section{Modified saturation "assumption"}

We consider the following model problem:

$$
\left\{\begin{aligned}
u & \in H_{0}^{1}(\Omega) \\
-\Delta u & =f \text { in } \Omega
\end{aligned}\right.
$$

where $\Omega$ is a bounded polygonal domain in $R^{2}$ and $f \in L^{2}(\Omega)$. Let $T_{h}$ be a conforming and shape regular triangulation by triangular elements, we set

$$
\begin{array}{ll}
V_{1}=\left\{v_{h} \in H_{0}^{1}(\Omega) ;\right. & \left.\forall T \in T_{h}, v_{h \mid T} \in P_{1}(T)\right\}, \\
V_{2}=\left\{v_{h} \in H_{0}^{1}(\Omega) ;\right. & \left.\forall T \in T_{h}, v_{h \mid T} \in P_{2}(T)+\operatorname{span}\left\{b_{T}\right\}\right\},
\end{array}
$$

where $b_{T}$ is the cubic bubble function, and consider the following problems $(k=1,2)$ :

$$
\left\{\begin{array}{l}
\text { Find } u_{k} \in V_{k} \\
\forall v_{k} \in V_{k} ; \quad \int_{\Omega} \nabla u_{k} \cdot \nabla v_{k} d x=\int_{\Omega} f v_{k} d x .
\end{array}\right.
$$

In the sequel, we denote by $C$ the positive generic constant independent of the mesh size which can change from one line to another.

Theorem 2.1. There exists a constant $\alpha \in[0,1[$ depending on the minimum angles of $T_{h}$ so that

$$
\left|u-u_{2}\right|_{1, \Omega} \leqslant \alpha\left|u-u_{1}\right|_{1, \Omega}+C\left(\sum_{T \in T_{h}} h_{T}^{2}\left\|f-f_{T}\right\|_{0, T}^{2}\right)^{1 / 2},
$$

where $f_{T}=\frac{1}{\operatorname{meas}(T)} \int_{T} f d x$.

Proof. First, we introduce the operator $\Pi^{0}$ defined from $H^{1}(\Omega)$ onto $V_{2}$ by : for all $v \in H^{1}(\Omega), \Pi^{0} v$ is the unique element of $V_{2}$ such that

$$
\text { for all nodes } a \text { of } T_{h}, \quad \Pi^{0} v(a)=0,
$$

$$
\text { for all edges } e, \int_{e}\left(\Pi^{0} v-v\right) d \sigma=0 ; \text { for all } T \in T_{h}, \int_{T}\left(\Pi^{0} v-v\right) d x=0 .
$$

Using the Green formula, we have

$$
\left\{\begin{array}{c}
\forall T \in T_{h}, \quad \forall w_{h} \in P_{1}(T), \quad \forall v \in H^{1}(T), \\
\int_{T} \nabla w_{h} \nabla\left(v-\Pi^{0} v\right) d x=\int_{\partial T} \frac{\partial w_{h}}{\partial n_{T}}\left(v-\Pi^{0} v\right) d \sigma=0,
\end{array}\right.
$$

and by the scaling arguments and the trace theorem it is easy to prove that

$$
\forall v \in H^{1}(\Omega), \quad \forall T \in T_{h}, \quad\left|\Pi^{0} v\right|_{1, T} \leqslant C\left(h_{T}^{-1}\|v\|_{0, T}+|v|_{1, T}\right) .
$$


We now set $e_{k}=u_{k}-u, k=1,2$. We have for all $e_{h} \in V_{1}$ :

$$
\begin{aligned}
\left|u-u_{1}\right|_{1, \Omega}^{2}= & \int_{\Omega} \nabla u_{1} \nabla e_{1} d x-\int_{\Omega} f e_{1} d x \\
= & \int_{\Omega} \nabla u_{1} \nabla\left(e_{1}-e_{h}\right) d x-\int_{\Omega} f\left(e_{1}-e_{h}\right) d x \\
= & \int_{\Omega} \nabla u_{1} \nabla \Pi^{0}\left(e_{1}-e_{h}\right) d x-\int_{\Omega} f\left(e_{1}-e_{h}\right) d x \\
= & \int_{\Omega} \nabla\left(u_{1}-u_{2}\right) \nabla \Pi^{0}\left(e_{1}-e_{h}\right) d x+\int_{\Omega} f\left(\Pi^{0}\left(e_{1}-e_{h}\right)-e_{1}+e_{h}\right) d x \\
= & \int_{\Omega} \nabla\left(u_{1}-u_{2}\right) \nabla \Pi^{0}\left(e_{1}-e_{h}\right) d x+\int_{\Omega}\left(f-f_{T}\right)\left(\Pi^{0}\left(e_{1}-e_{h}\right)-e_{1}+e_{h}\right) d x \\
\leqslant & \left|u_{1}-u_{2}\right|_{1, \Omega}\left|\Pi^{0}\left(e_{1}-e_{h}\right)\right|_{1, \Omega} \\
& +C\left(\sum_{T \in T_{h}} h_{T}^{2}\left\|f-f_{T}\right\|_{0, T}^{2}\right)^{1 / 2}\left(\left|\Pi^{0}\left(e_{1}-e_{h}\right)\right|_{1, \Omega}+\left|e_{1}-e_{h}\right|_{1, \Omega}\right) \\
\leqslant & C\left(\left|u_{1}-u_{2}\right|_{1, \Omega}+\left(\sum_{T \in T_{h}} h_{T}^{2}\left\|f-f_{T}\right\|_{0, T}^{2}\right)^{1 / 2}\right) \\
& \times\left(\sum_{T \in T_{h}} h_{T}^{-2}\left\|e_{1}-e_{h}\right\|_{0, T}^{2}+\left|e_{1}-e_{h}\right|_{1, T}^{2}\right)^{1 / 2} .
\end{aligned}
$$

Then

$$
\begin{aligned}
\left|u-u_{1}\right|_{1, \Omega}^{2} \leqslant & C\left(\left|u_{1}-u_{2}\right|_{1, \Omega}+\left(\sum_{T \in T_{h}} h_{T}^{2}\left\|f-f_{T}\right\|_{0, T}^{2}\right)^{1 / 2}\right) \\
& \times \inf _{v_{h} \in V_{1}}\left(\sum_{T \in T_{h}} h_{T}^{-2}\left\|e_{1}-v_{h}\right\|_{0, T}^{2}+\left|e_{1}-v_{h}\right|_{1, T}^{2}\right)^{1 / 2} \\
\leqslant & C\left(\left|u_{1}-u_{2}\right|_{1, \Omega}+\left(\sum_{T \in T_{h}} h_{T}^{2}\left\|f-f_{T}\right\|_{0, T}^{2}\right)^{1 / 2}\right)\left|u-u_{1}\right|_{1, \Omega},
\end{aligned}
$$

we deduce that

$$
\left|u-u_{1}\right|_{1, \Omega} \leqslant C\left|u_{1}-u_{2}\right|_{1, \Omega}+C\left(\sum_{T \in T_{h}} h_{T}^{2}\left\|f-f_{T}\right\|_{0, T}^{2}\right)^{1 / 2} .
$$

Now, using the equality

$$
\left|u-u_{1}\right|_{1, \Omega}^{2}=\left|u-u_{2}\right|_{1, \Omega}^{2}+\left|u_{1}-u_{2}\right|_{1, \Omega}^{2}
$$


and since we can choose $C \geqslant 1$, we obtain

$$
\begin{aligned}
\left|u-u_{2}\right|_{1, \Omega}^{2} & \leqslant\left(1-C^{-1}\right)\left|u-u_{1}\right|_{1, \Omega}^{2}+C\left(\sum_{T \in T_{h}} h_{T}^{2}\left\|f-f_{T}\right\|_{0, T}^{2}\right)^{1 / 2} \\
& =\alpha^{2}\left|u-u_{1}\right|_{1, \Omega}^{2}+C\left(\sum_{T \in T_{h}} h_{T}^{2}\left\|f-f_{T}\right\|_{0, T}^{2}\right)^{1 / 2} .
\end{aligned}
$$

with $\alpha \in[0,1[$.

\section{Hierarchical a posteriori error estimators}

In this section, we are interested in the hierarchical a posteriori error estimator. From the previous results we have

$$
\left|u_{1}-u_{2}\right|_{1, \Omega} \leqslant\left|u-u_{1}\right|_{1, \Omega} \leqslant C\left\{\left|u_{1}-u_{2}\right|_{1, \Omega}+\left(\sum_{T \in T_{h}} h_{T}^{2}\left\|f-f_{T}\right\|_{1, T}^{2}\right)^{1 / 2}\right\} .
$$

Since $u_{2}$ is expensive to compute, following Bank et al $[2,3]$, we introduce " hierarchical" space and solve a discrete problem with smaller dimensions than $V_{2}$. For this purpose we set

$$
V_{2}^{0}=\left\{v_{h} \in V_{2}, \text { so that for all nodes } a \text { of } T_{h}, v_{h}(a)=0\right\}
$$

and we introduce the discrete problem

$$
\left\{\begin{array}{l}
\text { Find } \quad u_{2}^{0} \in V_{2}^{0}, \\
\forall v_{2}^{0} \in V_{2}^{0} ; \quad \int_{\Omega} \nabla u_{2}^{0} \nabla v_{2}^{0} d x=\int_{\Omega} \nabla u_{1} \nabla v_{2}^{0} d x-\int_{\Omega} f v_{2}^{0} d x .
\end{array}\right.
$$

We have the following theorem.

Theorem 3.1. There exists a constant $C_{0}$ depending only on the minimum angle of $T_{h}$ such that

$$
\left|u_{2}^{0}\right|_{1, \Omega} \leqslant\left|u-u_{1}\right|_{1, \Omega} \leqslant C_{0}\left|u_{2}^{0}\right|_{1, \Omega}+C\left(\sum_{T \in T_{h}} h_{T}^{2}\left\|f-f_{T}\right\|_{1, T}^{2}\right)^{1 / 2}
$$

Proof. The first inequality is trivial. Let us prove the second one. Arguing as in the proof of Theorem 2.1, we can assume that

$$
f=f_{T} \quad \text { on } T, \quad \forall T \in \mathcal{T}_{h},
$$


Using the same notations as in the proof of the last theorem, we have for all $e_{h} \in V_{1}$ :

$$
\begin{aligned}
\left|u_{1}-u_{2}\right|_{1, \Omega}^{2} & =\int_{\Omega} \nabla u_{1} \nabla\left(u_{1}-u_{2}\right)-\int_{\Omega} f\left(u_{1}-u_{2}\right) d x \\
& =\int_{\Omega} \nabla u_{1} \nabla \Pi^{0}\left(u_{1}-u_{2}-e_{h}\right)-\int_{\Omega} f\left(u_{1}-u_{2}-e_{h}\right) d x \\
& =\int_{\Omega} \nabla u_{2}^{0} \nabla \Pi^{0}\left(u_{1}-u_{2}-e_{h}\right)-\int_{\Omega} f\left(u_{1}-u_{2}-e_{h}-\Pi^{0}\left(u_{1}-u_{2}-e_{h}\right)\right) d x \\
& =\int_{\Omega} \nabla u_{2}^{0} \nabla \Pi^{0}\left(u_{1}-u_{2}-e_{h}\right) \leqslant\left|u_{2}^{0}\right|_{1, \Omega}\left|\Pi^{0}\left(u_{1}-u_{2}-e_{h}\right)\right|_{1, \Omega},
\end{aligned}
$$

arguing as above, we obtain

$$
\left|u_{1}-u_{2}\right|_{1, \Omega} \leqslant C\left|u_{2}^{0}\right|_{1, \Omega}
$$

Combining this inequality with

$$
\left|u-u_{1}\right|_{1, \Omega} \leqslant C\left|u_{1}-u_{2}\right|_{1, \Omega}
$$

we obtain

$$
\left|u-u_{1}\right|_{1, \Omega} \leqslant C\left|u_{2}^{0}\right|_{1, \Omega}
$$

Now, we can introduce a local a posteriori error estimator. To do this, we begin with some notations. We denote by $E_{I}$ the set of interior edges for all $e \in E_{I}$, by $\phi_{e}$ - the canonical continuous piecewise quadratic basis function corresponding to the midpoint of the edge $e$, and, finally, by $b_{T}$ the bubble function corresponding to the triangle $T$ of $T_{h}$ is denoted. We set

$$
\begin{gathered}
\forall e \in E_{I}, \quad a_{e}=\frac{\int_{\Omega} \nabla u_{1} \nabla \phi_{e} d x-\int_{\Omega} f \phi_{e} d x}{\left|\phi_{e}\right|_{1, \Omega}^{2}}, \\
\forall T \in T_{h}, \quad c_{T}=\frac{\int_{\Omega} \nabla u_{1} \nabla b_{T} d x-\int_{\Omega} f b_{T} d x}{\left|b_{T}\right|_{1, \Omega}^{2}}:=-\frac{1}{\left|b_{T}\right|_{1, \Omega}^{2}} \int_{T} f b_{T} d x
\end{gathered}
$$

and

$$
\epsilon=\sum_{e \in E_{I}} a_{e} \phi_{e}+\sum_{T \in T_{h}} c_{T} b_{T} \in V_{2}^{0} .
$$

We introduce the bilinear form $d(.,$.$) defined on \left(V_{2}^{0}\right)^{2}$ by

$$
\begin{aligned}
& \forall(v, w) \in\left(V_{2}^{0}\right)^{2} ; \quad v=\sum_{e \in E_{I}} v_{e} \phi_{e}+\sum_{T \in T_{h}} v_{T} b_{T}, \quad w=\sum_{e \in E_{I}} w_{e} \phi_{e}+\sum_{T \in T_{h}} w_{T} b_{T}, \\
& d(v, w):=\sum_{e \in E_{I}} v_{e} w_{e}\left|\phi_{e}\right|_{1, \Omega}^{2}+\sum_{T \in T_{h}} v_{T} w_{T}\left|b_{T}\right|_{1, \Omega}^{2} .
\end{aligned}
$$


It is clear that

$$
\forall v_{2}^{0} \in V_{2}^{0} ; \quad d\left(\epsilon, v_{2}^{0}\right)=\int_{\Omega} \nabla u_{1} \nabla v_{2}^{0} d x-\int_{\Omega} f v_{2}^{0} d x=\int_{\Omega} \nabla u_{2}^{0} \nabla v_{2}^{0} d x .
$$

We have the following theorem.

Theorem 3.2. There exist constants $C_{0}$ and $C_{1}$ depending only on the minimum angle of $T_{h}$ so that

$$
\left|u-u_{1}\right|_{1, \Omega} \leqslant C_{0}|\epsilon|_{1, \Omega}+C\left(\sum_{T \in T_{h}} h_{T}^{2}\left\|f-f_{T}\right\|_{1, T}^{2}\right)^{1 / 2}
$$

and

$$
\forall T \in T_{h}, \quad|\epsilon|_{1, T} \leqslant C_{1}\left|u-u_{1}\right|_{1, \Delta(T)},
$$

where $\Delta(T)$ is the set of elements sharing an edge with $T$.

Proof. First, using the definition and scaling arguments we have

$$
\left|u_{2}^{0}\right|_{1, \Omega}^{2}=\int_{\Omega} \nabla u_{1} \nabla u_{2}^{0} d x-\int_{\Omega} f u_{2}^{0} d x=d\left(\epsilon, u_{2}^{0}\right) \leqslant C\left|u_{2}^{0}\right|_{1, \Omega}|\epsilon|_{1, \Omega} .
$$

Therefore, using Theorem 3.1, we have

$$
\left|u-u_{1}\right|_{1, \Omega} \leqslant C_{0}|\epsilon|_{1, \Omega}+C\left(\sum_{T \in T_{h}} h_{T}^{2}\left\|f-f_{T}\right\|_{1, T}^{2}\right)^{1 / 2} .
$$

As for the lower bound of the error, let $T \in T_{h}$, for each edge $e \in E_{I}$ of $T$, since $\left|\phi_{e}\right|_{1, \Omega} \geqslant C$ and $\operatorname{supp}\left(\phi_{e}\right) \subset \Delta(T)$, we have

$$
\left|a_{e}\right| \leqslant C\left|u-u_{1}\right|_{1, \Delta(T)} .
$$

Likewise, using the same arguments, we also have

$$
\left|c_{T}\right| \leqslant C\left|u-u_{1}\right|_{1, \Delta(T)}
$$

Using the last inequalities and the regularity of the mesh, we obtain

$$
|\epsilon|_{1, T} \leqslant \sum_{e \in \partial T}\left|a_{e}\right|\left|\phi_{e}\right|_{1, \Omega}+\left|c_{T}\right|\left|b_{T}\right|_{1, \Omega} \leqslant C\left|u-u_{1}\right|_{1, \Delta(T)} .
$$

\section{References}

[1] A. Agouzal and J. Olaiz, A posteriori error estimator on stars for nonconforming finite elements approximations, submitted to Numer. Math.

[2] R. E. Bank and A. Weiser, Some a posteriori error estimators for elliptic partial differential equations, Math. Comp., 44 (1985), pp. 283-301. 
[3] R. E. Bank and B. D. Welfert, A posteriori error estimates for the Stokes problem, SIAM J. Numer. Anal., 28 (1991), pp. 591-623.

[4] W. Dorfler and R. H. Nochetto, Small data oscillation implies the saturation assumption, to appear in Numer. Math.

[5] R. H. Nochetto, Removing the saturation assumption in a posteriori error analysis, Rend., Sci. Mat. Appl. A, 127 (1993), pp. 67-82. 